\title{
Some Insight into the Wind-Induced Vibration of Stay Cables in the Context of Rigid Static Inclined Circular Cylinder
}

\author{
A. Raeesi ${ }^{1 \dagger}$, S. Cheng ${ }^{2}$ and D.S-K. Ting ${ }^{3}$ \\ ${ }^{l}$ Graduates Student, Department of Civil and Environmental Engineering, University of Windsor \\ ${ }^{2}$ Associate professor, Department of Civil and Environmental Engineering, University of Windsor \\ ${ }^{3}$ Professor, Department of Mechanical, Automotive and Materials Engineering, University of Windsor \\ 401 Sunset Ave., Windsor, ON., Canada N9B 3P
}

†Corresponding AuthorEmail: raeesi@uwindsor.ca

(Received October 22, 2010; accepted April 4, 2011)

\begin{abstract}
Wind-induced cable vibration is a contemporary issue in cable-stayed bridges, which potentially threats the safety and durability of the structure. A thorough understanding of the fundamental physics underlying these phenomena is a priori for developing effective remedies to resolve the issue. In the present paper, possible mechanisms associated with two different types of wind-induced cable vibration phenomena have been studied based on a set of wind tunnel experimental data on a rigid circular cylinder. A number of analyses were applied to the unsteady surface pressure data sampled on the cylinder model to elucidate the possible mechanisms of these phenomena. Negative aerodynamic damping ratios were identified in the ranges of Reynolds number and cylinder orientation where divergent galloping type of response is expected to occur. A breakdown range of wind-cable relative angle was detected in which the regular Karman vortex shedding was suppressed within the subcritical Reynolds number range. In the critical Reynolds number range, however, the symmetry of surrounding flow field beyond this breakdown range could be altered drastically, leading to considerable changes in the lift force which is responsible for the negative aerodynamic damping ratio values. Significant increase of correlation length of sectional aerodynamic forces was also detected within this breakdown range in the critical regime. This, combined with the negative aerodynamic damping, is proposed to be a possible necessary onset condition for the galloping of dry inclined cables. The limited-amplitude instability, which occurred in the subcritical Re range, on the other hand, was found to be caused by the mitigation of regular Karman vortex shedding in the breakdown range while the spatial flow field was strongly correlated. In addition, the decay in correlation of aerodynamic forces in the critical Re range was believed to be key to the suppression of this unstable response.
\end{abstract}

Keywords: Circular cylinder; Dry inclined cable galloping; Critical Reynolds number regime; Spatial correlation; Aerodynamic damping; High-speed vortex excitation.

\section{NOMENCLATURE}

$\begin{array}{ll}\mathrm{C}_{\mathrm{L}} & \text { lift force coefficient } \\ \mathrm{C}_{\mathrm{D}} & \text { drag force coefficient } \\ \mathrm{L}_{\mathrm{C}} & \text { correlation length } \\ R_{i j} & \text { spatial correlation } \\ \mathrm{Re} & \text { Reynolds number } \\ \mathrm{U} & \text { mean wind speed } \\ \mathrm{U}_{\mathrm{N}} & \text { normal wind speed component } \\ \mathrm{U}_{\mathrm{R}} & \text { reduced velocity force } \\ m & \text { mass per unit length of cylinder } \\ \mathrm{z} & \text { axial distance on the cylinder model from } \\ \omega_{\mathrm{n}} & \text { undamped circular frequency of the cylinder } \\ & \text { model }\end{array}$

\section{INTRODUCTION}

Flow past a circular cylinder is a phenomenon commonly seen in daily life and in various engineering

\author{
midspan \\ $\alpha \quad$ cylinder model inclination angle \\ $\alpha_{\mathrm{f}} \quad$ cable inclination \\ $\beta \quad$ cylinder model yaw angle \\ $\beta_{\mathrm{f}} \quad$ cable yaw angle \\ $\gamma$ angle between major direction of cable motion \\ and $\mathrm{U}_{\mathrm{N}}$ \\ relative wind-cable angle \\ cylinder model circumferential angle \\ aerodynamic damping ratio
}

applications, such as wind past stay cables on cablestayed bridges and ocean current past marine guy cables. Due to interaction between the structure and the passing flow, various flow-induced vibration 
phenomena may occur. The wind-induced vibrations of bridge stay cables reported from many bridge sites in recent years is a typical example (Hikami and Shiraishi, 1988; Irwin et al, 1999; Main and Jones, 2001; Bosch, 2006). Based on the conditions of wind, precipitation, and stay cable (physical properties and geometrical layout), it was observed that a cable could exhibit different response characteristics, some of which are accompanied with fairly large amplitude and therefore cause serious concerns in the design of cable-stayed bridges. While the subject of normal flow past a circular cylinder has been extensively explored for over a century, that of an inclined circular cylinder appears to lack behind. In particular, this type of engineering problem is extremely rich in fundamental fluid dynamics phenomena which are yet to be discovered. A good appreciation of the physics underlying them is a priori to unveiling the mechanisms of a broad range of flow-induced vibrations observed on structures.

In the case of a bridge stay cable, the oncoming wind may or may not be perpendicular to the cable. Fig. 1a portrays part of the superstructure of a typical cablestayed bridge with two parallel cable planes. Shown in the figure is a stay cable in one of the cable planes, with one end anchored on the bridge deck and the other one on the pylon shaft. As can be seen from the figure, the relative angle $\varphi$ between the cable axis $A B$ and the oncoming wind direction $U$ is determined by two independent cable orientation angles, i.e. the inclination angle $\alpha_{\mathrm{f}}$, and the yaw angle $\beta_{\mathrm{f}}$ by:

$\cos \phi=\cos \alpha_{f} \cdot \cos \beta_{f}$

where the inclination angle $\alpha_{\mathrm{f}}$ is the angle between the cable axis (along $\mathrm{AB}$ direction) and its horizontal projection $\mathrm{OA}$, and the yaw angle $\beta_{\mathrm{f}}$ is the angle between the wind direction and the cable horizontal projection (along OA direction). The subscript " $\mathrm{f}$ " in $\alpha_{\mathrm{f}}$ and $\beta_{\mathrm{f}}$ signifies that these orientation angles correspond to a "full-scale cable" in real bridges. When wind blows past a cable-stayed bridge, the cable-wind relative angle $\varphi$ plays an important role in wind-induced cable vibrations. It is a common practice in wind tunnel studies to consider the cable as a rigid circular cylinder which is elastically supported at its two ends. Therefore, flow past a stay cable could be investigated in the context of flow past a rigid inclined and/or yawed circular cylinder in the wind tunnel (e.g. Matsumoto et al. 1990; Irwin et al. 1999; Zuo and Jones 2009).

There have been a number of studies directly dedicated to the aerodynamic aspects of bridge stay cables and their excitation mechanisms. The various wind-induced cable vibration phenomena observed either on bridge sites or in wind tunnel tests include rain-wind induced vibration (Hikami and Shiraishi 1988; Honda et al. 1995; Matsumoto et al. 1998), dry-inclined cable galloping (Miyata et al. 1994; Saito et al. 1994; Honda et al. 1995; Cheng et al. 2008a, 2008b), and high-speed vortex induced vibration (Matsumoto 1998; Matsumoto et al. 1999, 2007a; Cheng et al. 2008a, 2008b). Despite its complexity, the extensive research effort in recent years appears to make some progress in road on the mechanisms associated with the rain-wind induced vibrations, of which the wind velocity, precipitation intensity, cable orientation and surface conditions are identified as key parameters. The latter two aerodynamic responses are observed in the absence of precipitation. Their excitation mechanisms and the possible relations between them are still not conclusive.
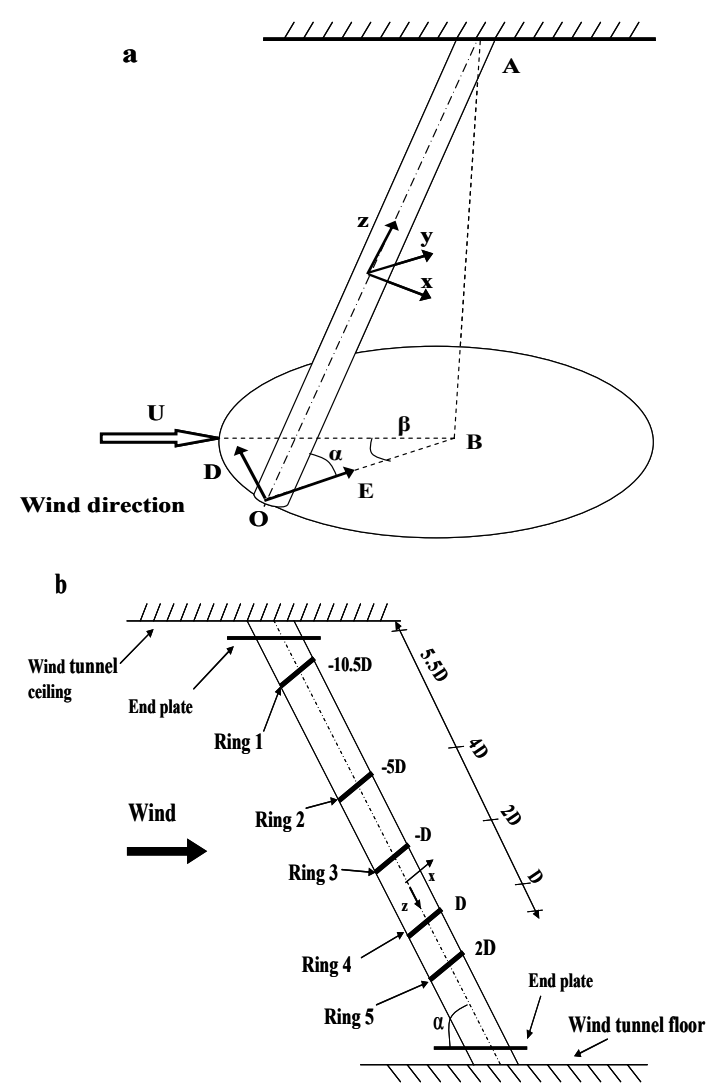

Fig. 1. The angle definitions and directions of motion in the normal plane; a) the definition of angles on the bridge site; b) major direction of motion on the cross section of cable

Shirakashi et al. (1986) were perhaps the first who noted the role of axial flow along an inclined circular cylinder model on disturbing regular Karman vortex shedding. It was subsequently confirmed by Matsumoto et al. (1990). They also proposed that the role of the secondary axial flow in aerodynamic excitation of a cylinder model was similar to an "air-curtain". It would interrupt the fluid interaction between the two separated shear layers and generate an unsteady inner-circulatory flow at either the lower-leeward or the upper-leeward circumferential surface of the cylinder, leading to a region of significantly lower surface pressure which could result in higher oscillatory aerodynamic forces and consequently, violent vibration of inclined cylinder model. Later, it was also noted (Matsumoto 1998; Matsumoto et al. 1999) that the interaction between conventional "Karman vortex shedding" and the socalled axial vortex shedding, with the frequency of the latter being about one-third of the former, could be the contributor to the high-speed vortex excitation. This implies that the cylinder response would be amplified when the axial vortices shed once every three Karman vortices from either side of the cylinder model. In their persisting effort to reveal the mechanisms of windinduced inclined cable vibrations, Matsumoto et al. (2007a; 2007b) also pointed out the impact of 
mitigating Karman vortex shedding on the onset of selfexcited cable vibrations. Based on such effect, it was proposed that the secondary axial flow could be fundamental to the vibration mechanism of inclined cables.

The divergent motion in the absence of rain, which is known as dry inclined cable galloping, was observed in a number of wind tunnel studies such as Saito et al. (1994) and Honda et al. (1995) in the subcritical Re regime; and Miyata et al. (1994) and Cheng et al. $(2003,2008 \mathrm{a}, 2008 \mathrm{~b})$ in the transition and critical Reynolds number regime. It was found that when such kind of aerodynamic instability occurred on an inclined stay cable, the flow within the critical Reynolds number range was spatially better correlated, which corresponded to larger aerodynamic forces acting on the cylinder (Cheng and Tanaka, 2005). The same phenomenon has been observed on a cross-flow circular cylinder (Raeesi et al. 2008). A further study (Cheng et al. 2008a) proposed that the classical Den Hartog criterion for iced-cable galloping was applicable to explain the excitation mechanisms.

To fully disclose the physics behind various windinduced vibrations of bridge stay cables, in particular, those occurred without rain, fundamental studies on the behavior of an inclined circular cylinder in flow are essential. It is obvious from the above reviewed literatures that there were only a few studies dedicated to elucidate the physics underlying those phenomena, and those were mostly conducted within the subcritical Reynolds number range. There is a serious lack of fundamental fluid dynamic studies concerning the impact of critical regime on the wind-induced vibration phenomena of bridge stay cables. The present study aims at studying the possible excitation mechanisms of dry inclined cable galloping and high-speed vortex excitation using a set of wind tunnel data on a rigid circular cylinder. The aerodynamic damping of the cylinder model, spatial correlation and power spectra of aerodynamic forces under different combinations of wind and cable conditions will be analyzed.

\section{EXPERIMENTAL DETAILS AND REMARKS ON AN EARLIER STUDY}

A series of static and dynamic wind tunnel tests on an inclined circular cylinder were conducted at the National Research Council of Canada by one of the authors (Cheng and Tanaka, 2005; Cheng et al. 2008b). The key motivation for this set of experimental work was to investigate the possible mechanisms associated with wind-induced vibration of bridge stay cables under no precipitation conditions. The aerodynamic instabilities of cables observed in some earlier experimental work were successfully reproduced in the dynamic tests, to confirm and verify the onset physical conditions. Subsequently, surface pressure of a rigid steel circular cylinder model was measured in the static tests. The cylinder was placed at different orientations equivalent to those where unstable cable responses were observed in the dynamic tests. The experimental setups of static and dynamic tests are described in details by Cheng et al. (2008b). The testing conditions are summarized in Table 1. In both tests, despite the size limitations of wind tunnel facilities and the difference in model support conditions, the relative angle $\phi$ between the cylinder model axis and the wind direction, as well as the motion direction of model were kept consistent with those in the simulated full-scale cases on real bridges. Details of the transformation of fullscale cable orientations to wind tunnel model setups are explained by Cheng et al. (2008b). Direction of motion and those of positive lift and drag forces in the plane normal to the cylinder axis are shown in Fig. 1b, where $\gamma$ is the angle between the major direction of cable motion and $U_{N}$, which is the wind velocity component normal to the cable axis. In the current study, this angle is determined based on the angle relations derived in Cheng et al. (2008b).

It is a common practice to decompose the response of bridge stay cables into in-plane and out-of-plane components. The in-plane motion occurs within the plane of cable axis and its horizontal projection and is normal to the cable axis, i.e. coincides with vector $\mathrm{AE}$ in Fig. 1a. The out-of-plane component, however, is normal to the cable plane. It is represented by vector $\mathrm{AD}$ in Fig. 1a, which is normal to plane BOA. The terms "in-plane" and "out-of-plane" will be used in the present paper to refer the two cable response components. If wind blows along an inclined but nonyawed cable (i.e. $\alpha_{\mathrm{f}} \neq 0^{\circ}, \beta_{\mathrm{f}}=0^{\circ}$ ), the major direction of cable response would be out-of-plane (i.e. along $\mathrm{AD}$ in Fig. 1a and lift direction in Fig. 1b) and thus $\gamma=90^{\circ}$. This means the cable is solely excited by the lift force. When wind is skewed with respect to the cable (i.e. $\alpha_{\mathrm{f}} \neq 0^{\circ}, \quad \beta_{\mathrm{f}} \neq 0^{\circ}$, both in-plane and out-of-plane components would appear in the motion trajectory typically with one of them being dominant (Cheng et al. 2008b). However, when $\beta_{\mathrm{f}}=90^{\circ}$ and hence $\varphi=90^{\circ}$ (cylinder in cross-flow case), the response would be purely in-plane, i.e. along $\mathrm{AE}$ direction within BOA plane in Fig. 1a or along lift direction in Fig. 1b.

Two different types of response were observed in the dynamic tests (Cheng et al. 2008b). Each type of unstable response differed in terms of amplitude of vibrations, dominant direction of motion, and Reynolds number range. Since one of the focal points in the present work is to investigate and compare aerodynamic properties and surrounding flow patterns of a cylinder model with different spatial orientations when exposed to the same oncoming flow condition, the definition of Reynolds number in this paper is the nominal Reynolds number based on free stream velocity $U$ and the cylinder diameter $\mathrm{D}$, i.e. $\operatorname{Re}=\mathrm{UD} / v$; where $v$ is the kinematic viscosity.

The divergent type of motion with increasing amplitude (until it was suppressed manually) was observed in an experimental setup corresponding to a full-scale cable inclined and yawed both at $45^{\circ}$ with respect to the wind $\left(\alpha_{\mathrm{f}}=\beta_{\mathrm{f}}=45^{\circ}\right.$, thus $\left.\varphi=60^{\circ}\right)$. While the dominating motion was in the in-plane direction, the out-of-plane component was also significant. Consequently, an elliptical trajectory was formed with its major axis at $\gamma=54.7^{\circ}$ (Cheng et al., 2008b). Such a divergent type of response on an inclined and yawed cable was also observed in the wind tunnel studies by Miyata et al. (1994), Saito et al. (1994), and Honda et al. (1995). 
Table 1 Testing conditions of wind tunnel experiments by Cheng et al. (2008b)

\begin{tabular}{|c|c|c|c|c|c|c|}
\hline Test & $\begin{array}{c}\text { Parameter } \\
\text { measured }\end{array}$ & $\begin{array}{c}\text { Model diameter } \\
(\mathrm{mm})\end{array}$ & $\begin{array}{c}\text { Model aspect } \\
\text { ratio }\end{array}$ & $\phi$ range $\left(^{\circ}\right)$ & $\begin{array}{c}\text { Wind speed } \\
(\mathrm{m} / \mathrm{s})\end{array}$ & $\begin{array}{c}\text { Re range } \\
\left(\times 10^{5}\right)\end{array}$ \\
\hline Dynamic & displacement & 160 & $\approx 40$ & $35-60$ & $10-36$ & $1-3.4$ \\
\hline Static & pressure & 88.9 & $\approx 22-27$ & $54.7-107$ & $10-95$ & $0.8-6$ \\
\hline
\end{tabular}

Table 2 Response characteristics of the two observed critical cases

\begin{tabular}{|c|c|c|c|c|c|c|c|c|c|}
\hline \multirow[t]{2}{*}{ Setup } & \multirow[t]{2}{*}{$\begin{array}{l}\text { Response } \\
\text { type }\end{array}$} & \multicolumn{2}{|c|}{$\begin{array}{c}\text { Full-Scale cable } \\
\text { angles }\left({ }^{\circ}\right)\end{array}$} & \multirow{2}{*}{$\begin{array}{c}\begin{array}{c}\text { Cable- } \\
\text { wind } \\
\text { relative } \\
\text { angle }\left(^{\circ}\right)\end{array} \\
\phi\end{array}$} & \multirow{2}{*}{$\begin{array}{c}\begin{array}{c}\text { Major } \\
\text { motion } \\
\text { direction }\left(^{\circ}\right)\end{array} \\
\gamma \gamma\end{array}$} & \multicolumn{2}{|c|}{$\begin{array}{l}\text { Static wind } \\
\text { tunnel } \\
\text { orientation } \\
\text { angles }\left({ }^{\circ}\right) \\
\end{array}$} & \multicolumn{2}{|c|}{ Unstable Range } \\
\hline & & $\alpha_{\mathrm{f}}$ & $\beta_{\mathrm{f}}$ & & & $\alpha$ & $\beta$ & $\operatorname{Re}\left(\times 10^{5}\right)$ & $\mathrm{U}_{\mathrm{r}}^{*}$ \\
\hline $2 \mathrm{C}$ & Divergent & 45 & 45 & 60 & 54.7 & 54.7 & 30 & $3.16-3.37$ & $134-143$ \\
\hline $2 \mathrm{~A}$ & $\begin{array}{c}\text { Limited } \\
\text { amplitude }\end{array}$ & 60 & 0 & 60 & 90 & 60 & 0 & $1.86-1.96$ & $79-84$ \\
\hline
\end{tabular}

$* \mathrm{U}_{\mathrm{r}}=\mathrm{U} / \mathrm{f} \cdot \mathrm{D}$

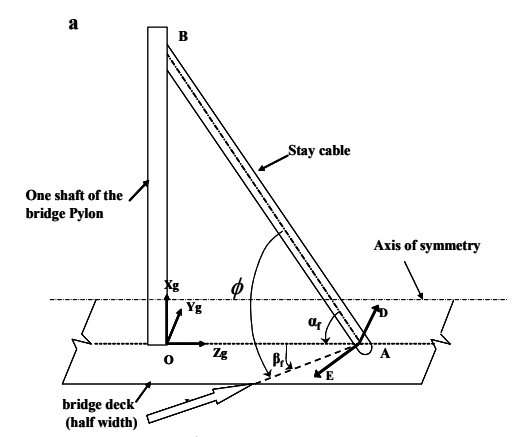

$\mathbf{b}$
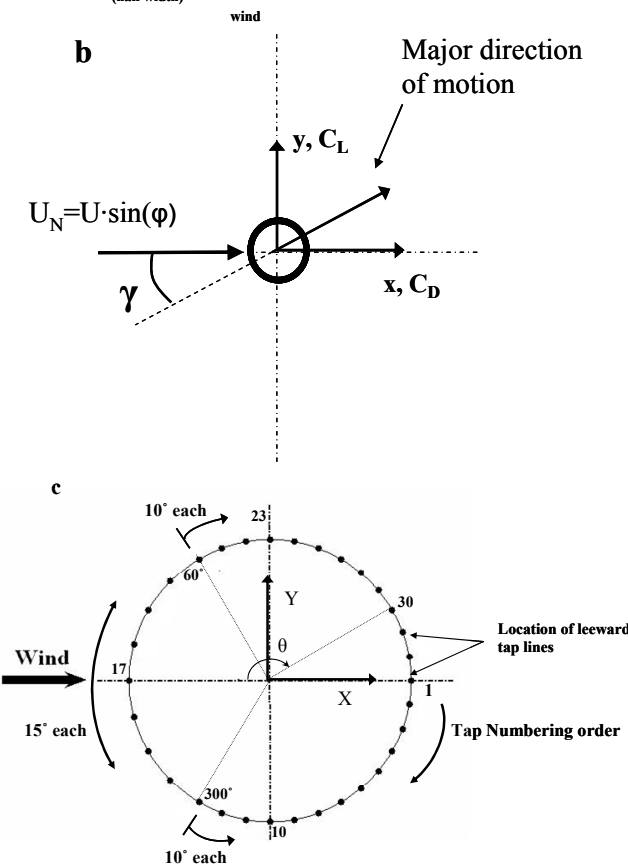

Fig. 2. Experimental setup of static model test; a)

Definition of orientation angles; b) Arrangement of pressure tap ring along the cylinder span (when $\alpha=90^{\circ}$ );

c) Array of pressure taps on a typical ring
The surface conditions of the cylinder models in these four tests were all reported to be "smooth". Since this divergent type response occurred without the presence of precipitation and had similar characteristics as galloping, it was thus called "dry inclined cable galloping" in the subsequent studies. When it occurred, the cable model orientation in the tests by Miyata et al. (1994) was identical to that by Cheng et al. (2008b), while those in the tests by Saito et al. (1994) and Honda et al. (1995) corresponded to a non-yawed full-scale cable inclined at $45^{\circ}$ to the wind. This would result in a relative cable-wind angle $\varphi$ of $45^{\circ}$. The circular cylinder models used in all these studies had diameters of typical bridge stay cables but differed in terms of Reynolds number range, length, and unit mass. As for Reynolds number, the divergent motion was detected in the subcritical (Saito et al. 1994; Honda et al. 1995), transition (Miyata et al. 1994), and critical Reynolds number range (Cheng et al. 2008b). This suggests that the divergent type response or the so-called dry inclined cable galloping is very sensitive to the onset conditions and it can be triggered over a broad Reynolds number range. Although there is no specific field report of this phenomenon yet, there are a few reports form bridge site (Virlogeux 1998; Irwin et al. 1999) which seem to be more consistent with this explanation rather than rain-wind-induced vibration. Therefore, the possibility of such divergent response should not be disregarded and its onset conditions need to be clarified.

The second type of response, which had limited amplitude, was observed in a number of different setups. The most violent one occurred at a setup equivalent to a non-yawed full-scale cable inclined at $60^{\circ}$ with respect to the oncoming wind $\left(\alpha_{\mathrm{f}}=60^{\circ}, \beta_{\mathrm{f}}=0^{\circ}\right.$, thus $\varphi=60^{\circ}$ ). It was found to be rather easily suppressed by increasing the structural damping (Cheng et al. 2003). This type of limited-amplitude response was more similar to that observed by Matsumoto (1998) and Matsumoto et al. (1999 \& 2007a). The characteristics and critical onset conditions of the two types of 
responses identified in the dynamic tests and their equivalent static tests orientations are summarized in Table 2.

Static model tests were then designed to investigate the possible mechanisms associated with the two different types of response observed in the dynamic tests. Unsteady surface pressure measurements were carried out at five axial locations along the span of a rigid circular cylinder model. Fig. 2a schematically shows the cylinder setup in the static wind tunnel tests, with $\alpha$ and $\beta$ respectively as the inclination and yaw angle of the cylinder model with respect to the wind. The model inclination angle $\alpha$ is defined as the angle between the model axis and its horizontal projection on the wind tunnel floor, whereas the yaw angle $\beta$ is the angle between the horizontal projection of the cable model and the oncoming wind direction. As shown in Figs. 2b and $2 \mathrm{c}$, five pressure tap rings were arranged along the cylinder model with 32 pressure taps installed on each ring along the circumferential direction $\theta$. Previous analyses (Raeesi et al. 2008), however, showed that some data sampled at $\mathrm{z} / \mathrm{D}=-10.5$ (Ring 1 ) were slightly distorted, presumably due to its proximity to the end plate. A recent wind tunnel study (Yagi et al. 2009) also concluded that the existence of end plate on the upstream side of the cylinder model could affect model surface pressure extensively. Therefore, only data measured at the remaining four locations are used for further analysis in this paper. Tests were conducted under three different cylinder inclination angles of $54.7^{\circ}, 60^{\circ}$ and $90^{\circ}$, with a yaw angle sweep of $0^{\circ} \sim 120^{\circ}$ for the first two inclination angles. The $90^{\circ}$ inclination angle case corresponds to the classical problem of cross-flow past a circular cylinder.

Data in Table 2 shows that though the relative cablewind angle $\varphi$ in Setups $2 \mathrm{~A}$ and $2 \mathrm{C}$ are both $60^{\circ}$, the observed response characteristics and onset conditions are very different. This needs to be further studied from a more fundamental fluid dynamics point of view. The focus of the present paper is thus to utilize the unsteady surface pressure data from the static tests to explore the difference in the possible mechanisms and onset conditions of the two types of unstable responses observed in the dynamic tests.

\section{DISCUSSION OF OBSERVED PhenOMENA}

In this section, the applicability of a general expression for estimating cable aerodynamic damping ratio proposed by Macdonald and Larose (2006) in explaining the onset of unstable responses observed in Setups 2C and 2A (Divergent and Limited Amplitude cases in Table 2) is investigated using the surface pressure data of rigid circular cylinder sampled in the static tests. Following that, the axial correlation and power spectra of lift and drag forces on the cylinder when it is oriented to represent Setups $2 \mathrm{C}$ and $2 \mathrm{~A}$ are discussed.

\subsection{Aerodynamic Damping}

\subsubsection{Application of a Generalized Aerodynamic Damping Ratio Expression}

When a structure is excited by wind, the total damping contained in the oscillating system includes two parts, i.e. the inherent structural damping and the aerodynamic damping contributed by surrounding flow. In the classical galloping theory, which was proposed by Den Hartog (1956), the structure is modeled as a single-degree-of-freedom system. The aerodynamic damping is then proportional to $\left(\partial C_{L} / \partial \alpha_{a}+C_{D}\right)$, where $\mathrm{C}_{\mathrm{L}}$ and $\mathrm{C}_{\mathrm{D}}$ are, respectively, the lift and drag force coefficients, and $\alpha_{a}$ is the wind angle of attack. For a structure that has a non-circular cross-section, negative slope of lift against wind angle of attack could exist at certain orientations and Reynolds numbers, resulting in negative aerodynamic damping. Once it overcomes the positive structural damping, the overall effective damping of the vibrating structure will become negative, and divergent motion will be initiated. This criterion well explained the necessary onset condition of galloping when a power transmission line is coated with ice in winter. Its applicability in explaining the divergent motion observed in Setup 2C was investigated and confirmed by Cheng et al. (2008a). However, it should be noted that the classical Den Hartog criterion is derived based on a single-degree-offreedom model, whereas the unstable motion observed in Setup 2C follows an elliptical trajectory, i.e. it contains both in-plane and out-of-plane components. Further, in the case of an inclined and/or yawed circular cylinder, the aerodynamic forces and surrounding flow patterns are extremely sensitive to the model orientation and Reynolds number, which are not considered in the formulation of the classical Den Hartog criterion.

A general expression of quasi-steady aerodynamic damping of an inclined and/or yawed cylinder with arbitrary cross-section was derived by Macdonald and Larose (2006), of which the contributions of lift, drag, Reynolds number, wind-cylinder relative angle, and cable motion direction were all considered in the formulation. It is applicable to flow-induced vibrations of cylinder along any direction. In the current study, to estimate the aerodynamic damping of the circular cylinder model when subjected to the physical conditions simulated in the static tests, this general expression can be simplified as :

$$
\begin{aligned}
\zeta_{a}= & \frac{\mu \operatorname{Re}}{4 m \omega_{n}} \cos \gamma\left\{\cos \gamma\left[C_{D}\left(2 \sin \phi+\frac{\tan ^{2} \gamma}{\sin \phi}\right)+\frac{\partial C_{D}}{\partial \operatorname{Re}} \operatorname{Re} \sin \phi+\frac{\partial C_{D}}{\partial \phi} \cos \phi\right]\right. \\
& \left. \pm \sin \gamma\left[C_{L}\left(2 \sin \phi-\frac{1}{\sin \phi}\right)+\frac{\partial C_{L}}{\partial \operatorname{Re}} \operatorname{Re} \sin \phi+\frac{\partial C_{L}}{\partial \phi} \cos \phi\right]\right\}
\end{aligned}
$$

where $\zeta_{\mathrm{a}}$ is the aerodynamic damping ratio, $\mu$ is the dynamic viscosity, $\omega_{\mathrm{n}}$ is the undamped circular frequency of the cylinder model, $m$ is the mass per unit length of cylinder, $\gamma$ is the angle between the major direction of motion and normal velocity component (Fig. 1b), and $\varphi$ is the wind-cylinder relative angle. The terms included inside the first and the second square brackets are referred to as the drag and the lift terms, respectively. Though it appears that the drag term is dependent on the cable-wind relative angle $\varphi$ and the major motion direction angle $\gamma$, it should be noted that these two angles are interdependent (Cheng et al. $2008 \mathrm{~b}$ ). The dual sign of the lift force term in the

\footnotetext{
* Please note that Macdonald and Larose (2006) used different nomenclatures in their study.
} 
equation is associated with the asymmetric flow patterns around the cylinder model when the flow enters the critical range. At this stage, a single separation bubble forms on one side of the cylinder surface. Depending on at which side it occurs, the lift force could be either positive or negative (see, for example, Schewe 1983 and Zdravkovich 1997).

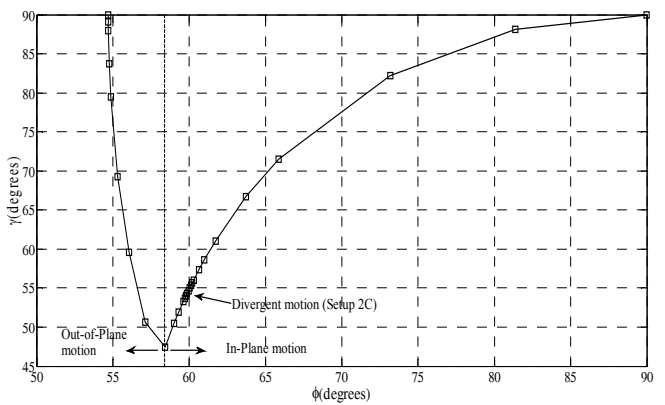

Fig. 3. Major direction of motion predicted for the range of $\phi$ in the static tests

Figure 3 portrays the relation between the windcylinder relative angle $\varphi$ and the cylinder major motion direction $\gamma$ based on the model orientations tested in the static experiment. The calculation of $\gamma$ was based on angle relations derived in Appendix of Cheng et al. (2008b). This means, for every specific orientation of the cylinder model in the static tests (combination of $\alpha$ and $\beta$ and thus $\varphi$ ) there would be an equivalent dynamic test setup in which the angle between $U_{N}$ and the major direction of motion (be it either in-plane or out-ofplane) is equal to the $\gamma$ portrayed in Fig. 3. Therefore, when $\gamma$ becomes $45^{\circ}$, both in-plane and out-of-plane direction would have equal contributions. The solid line in the figure corresponds to the cases when the model is inclined at $\alpha=54.7^{\circ}$ with a yaw sweep of $0^{\circ}$ to $120^{\circ}$; whereas the dashed line represents those of $\alpha=60^{\circ}$ and $\beta=0^{\circ}-120^{\circ}$. As can be observed from the figure, when $\alpha=54.7^{\circ}$, the range of $\gamma$, which represents the predominant motion direction, varies between $47.5^{\circ}$ and $90^{\circ}$. The minimum of $\gamma \approx 47.5^{\circ}$ occurs at $\varphi \approx 58.4^{\circ}$, which, according to Fig. 1b, lift and drag contributes almost equally toward excitation of the cable. For the divergent response observed in Setup $2 \mathrm{C}$, the major direction of motion is $\gamma \approx 54.7^{\circ}$. This value is close to $47.5^{\circ}$. Thus, both in-plane and out-of-plane components are significant. In the extreme case of non-yawed situation $\left(\alpha=54.7^{\circ}, \beta=0^{\circ}\right.$, and thus $\left.\varphi=54.7^{\circ}\right)$, wind blows along the cylinder, and the response will be cross-wind, i.e. out-of-plane with $\gamma=90^{\circ}$ along the direction of lift. This is represented by point B in Fig. 3 . In other words, as the wind-cable relative angle $\varphi$ decreases from $58.4^{\circ}$ to $54.7^{\circ}$, the out-of-plane component becomes progressively important in the cable vibration. On the contrary, if a cross-flow case is considered (point $C$ ), we have $\alpha=54.7^{\circ}$ and $\beta=90^{\circ}$; yielding a wind-cylinder relative angle of $\varphi=90^{\circ}$.

In this case, the cylinder would vibrate purely in plane along the lift direction. Thus, the angle represents the major motion direction is $\gamma=90^{\circ}$. So, when increasing the wind-cable relative angle $\varphi$ from $58.4^{\circ}$ to $90^{\circ}$, the cylinder motion becomes increasingly dominated by its in-plane component. Similar $\varphi-\gamma$ relation pattern can be observed for the dashed line, when the cylinder model is inclined by $60^{\circ}$ with a yaw sweep of $0^{\circ}$ to $120^{\circ}$. In this case, the minimum value of $\gamma=45^{\circ}$ occurs at $\varphi=69^{\circ}$. Again, this point distinguishes the importance of the out-of-plane component from the in-plane one in their contribution to the cylinder motion. For Setup 2A, of which the model is inclined at $60^{\circ}$ with no yaw, the relative angle is $\varphi=60^{\circ}$ and the motion direction is $\gamma=90^{\circ}$ (point B'). Therefore, the response of the cylinder model is purely out-of-plane. This agrees with the phenomenon observed in the dynamic tests (Cheng et al. 2008b).

To calculate aerodynamic damping of the cylinder model, the lift and drag coefficients obtained in the static tests are utilized in Eq. (2). Figure 4 shows the aerodynamic damping ratio of the model when $\alpha=54.7^{\circ}$ and $\beta=0^{\circ}-120^{\circ}$. Both positive and negative signs of the lift force term were considered and illustrated respectively in Figs. 4a and $4 b$. The "Re" and " $\varphi$ " axes in the figure are oriented in such a way that the results can be more clearly observed. The critical Reynolds number here is defined based on the detection of drag crisis when plotting the drag coefficient against Reynolds number. It varies depending on the cylinder model orientation with respect to wind. The critical Re regime corresponding to $65^{\circ}<\varphi<90^{\circ}$ is $2.95 \times 10^{5}<\operatorname{Re}<3.25 \times 10^{5}$, whereas that for $55^{\circ}<\varphi<65^{\circ}$ is $3.25 \times 10^{5}<\operatorname{Re}<3.55<10^{5}$. As can be seen from these two figures, in the subcritical Reynolds number range $\left(\operatorname{Re}<3 \times 10^{5}\right)$, aerodynamic damping ratio $\zeta_{\mathrm{a}}$ remains positive. Large negative aerodynamic damping ratios can be seen at $\varphi \approx 60^{\circ}$ (i.e. $\alpha=54.7^{\circ}, \beta \approx 30^{\circ}$ ) when $\operatorname{Re}>3 \times 10^{5}$. This corresponds to the critical onset condition of the divergent response observed in Setup $2 \mathrm{C}$.

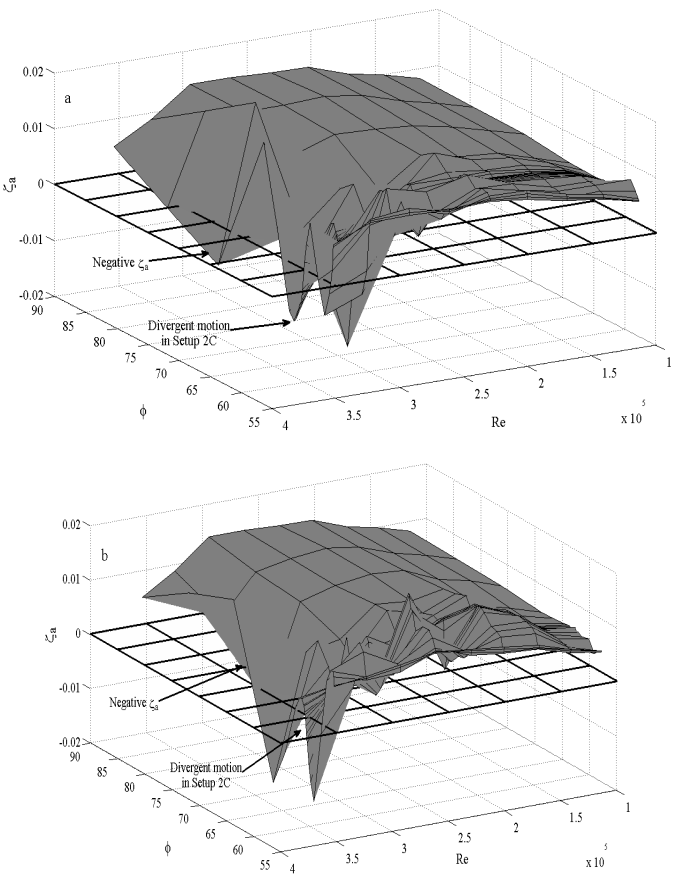

Fig. 4. Aerodynamic damping ratio of the cylinder in the static tests when model is inclined by $54.7^{\circ}$ with respect to wind, including Setup $2 \mathrm{C}\left(\phi=60^{\circ}, \gamma=54.7^{\circ}\right)$ condition; a) for $+\sin \gamma$ b) for $-\sin \gamma$ 
It is noticed in Fig. 4 that when $\operatorname{Re}>3 \times 10^{5}$, negative $\zeta_{\mathrm{a}}$ values can also be observed at $\varphi \approx 63^{\circ} \sim 81^{\circ}$. Within this range, the time-averaged non-zero lift force that already existed due to the cylinder orientation becomes relatively small, and the formation or burst of a single separation bubble may dramatically alter the magnitude of the lift force.

Thus, it seems that if the physical conditions of flow and cylinder satisfy this parameter range, the term $\partial \mathrm{C}_{\mathrm{L}} / \partial \mathrm{Re}$ in Eq. (2) has a more significant contribution to the negative value of $\zeta_{\mathrm{a}}$. Unfortunately, there are no available dynamic testing data to verify the occurrence of divergent motion in this range.

The aerodynamic damping ratio of cylinder in Setup 2A can be estimated by substituting $\gamma=90^{\circ}$ into Eq. (2),

$\zeta_{a}=\frac{\mu \operatorname{Re}}{4 m \omega_{n}}\left[\frac{1}{\sin \phi} C_{D}\right]$

This equation is valid when a non-yawed circular cylinder is inclined at an angle $\phi$ with respect to wind and vibrates in the cross wind direction. The aerodynamic damping ratio determined by Eq. (3) is always positive. This indicates that the limitedamplitude instability observed in Setup 2A would never become divergent. In other words, it is suggested by Eq. (3) that on a real cable-stayed bridge, when wind blows along the cable (i.e. $\beta_{\mathrm{f}}=0^{\circ}$ ), the response of the cable would not become divergent because aerodynamic damping remains positive. Therefore, the limited-amplitude instability which occurred in Setup $2 \mathrm{~A}$ does not have the same mechanism as that in Setup $2 \mathrm{C}$, but rather, it is associated with the existence of shear-layer instability which causes regular Karman vortex shedding and thus oscillatory lift force on a circular cylinder (see, for example, Snarski, 2004).

The form of Eq. (2) shows that when a cylinder is experiencing flow-induced vibration, besides properties of the passing flow and dynamic characteristics of the cylinder itself, the sense and magnitude of the aerodynamic damping ratio is also dependent on the orientation and response of the cylinder with respect to the flow, i.e., the cylinder-flow relative angle $\varphi$, and its major direction of motion, $\gamma$. Therefore, although the cylinder models in Setups $2 \mathrm{~A}$ and $2 \mathrm{C}$ have the same cable-wind relative angle of $\varphi=60^{\circ}$, due to the difference in the predominant direction of response, the aerodynamic damping ratio in Setup $2 \mathrm{C}$ is determined to be negative, which results in divergent galloping type response, whereas that in Setup 2A is positive, and thus the observed instability is limited in amplitude. In addition, results presented in Fig.4 clearly indicate that the galloping like motion occurred in Setup 2C can be successfully predicted from the negative aerodynamic damping ratio obtained from Eq.(2) under the combination of appropriate set of physical conditions, i.e. $\varphi=60^{\circ}$ and $\mathrm{Re}=3.16 \times 10^{5}$.

\subsubsection{Contribution to Negative Aerodynamic Damping Ratio from Different Terms}

In order to investigate the flow structure around the cylinder within the range of observed negative aerodynamic damping ratio seen in Fig. 4, circumferential surface pressure distribution around the cylinder at different axial locations of $\mathrm{z} / \mathrm{D}=-5,-1,1,2$ (Rings 2-5 as shown in Fig. 2b) were first averaged and then plotted against Re for different yaw angles in Fig. 5. It could be seen from Fig. 5a that at $\mathrm{Re}=3.25 \times 10^{5}$, when $\beta<40^{\circ} \quad\left(\varphi<63.7^{\circ}\right)$, pressure distribution is fairly symmetric and pressure coefficient $C_{P}$ values are relatively low.
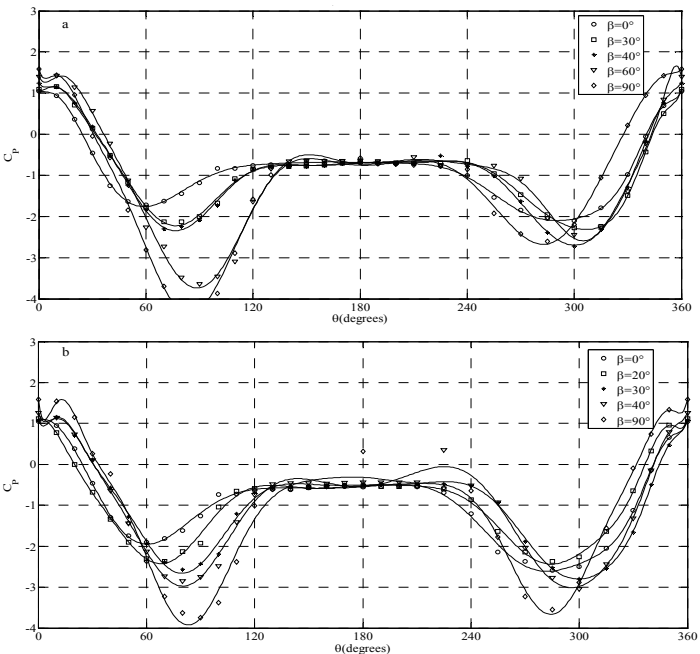

Fig. 5. Circumferential pressure distribution in the static tests when the model is inclined $54.7^{\circ}$ with respect to

the wind, including Setup $2 \mathrm{C}\left(\phi=60^{\circ}, \gamma=54.7^{\circ}\right)$ condition; a) $\operatorname{Re}=3.25 \times 10^{5}$ b) $\mathrm{Re}=3.55 \times 10^{5}$

The slight asymmetry in this range seems to be mostly due to the asymmetric orientation of the cylinder. However, when $\beta$ is increased to $60^{\circ}\left(\varphi=75.5^{\circ}\right)$, pressure distribution becomes clearly asymmetric and the asymmetry is strengthened with further increase of yaw angle to $\beta=90^{\circ}\left(\varphi=90^{\circ}\right)$. This suggests that at this Reynolds number $\left(\operatorname{Re}=3.25 \times 10^{5}\right)$, the transition to the critical regime occurs when increasing the cylinder yaw angle. At a higher Reynolds number of $3.55 \times 10^{5}$, as shown in Fig. 5(b), lower $C_{P}$ values at one side of the cylinder when $\beta=0^{\circ}\left(\varphi=54.7^{\circ}\right)$ indicates the formation of a single separation bubble. This regime is accompanied by clearly asymmetric circumferential pressure distribution. When increasing $\beta$ to $30^{\circ}(\varphi$ $=60^{\circ}$ ), however, the symmetric pattern reappears with another sharp drop of $C_{P}$ value on the other side of the cylinder. The reoccurrence of symmetric pattern is more obvious for $\beta>40^{\circ}\left(\varphi>63.7^{\circ}\right)$. As the cylinder becomes more yawed against the oncoming flow, the cylinder cross section "seen" by the flow varies from an elliptical shape to a more circular one, which would affect the effective Reynolds number and this may also have some influence on the transition from a single separation bubble regime to double separation bubble regime. This, along with the enhanced axial flow, could lead to a significant negative value of $\partial \mathrm{C}_{\mathrm{L}} / \partial \varphi$ term.

It is interesting to note that the physical conditions at which asymmetric surface pressure distribution is observed are consistent with those where negative aerodynamic damping is identified in Fig. 4. This set of 
results suggests that at $\mathrm{Re}>3 \times 10^{5}$, the range of cablewind relative angle of $\varphi \approx 55^{\circ} \sim 64^{\circ}\left(\beta=0^{\circ} \sim 40^{\circ}\right.$, while $\alpha=54.7^{\circ}$ ) is a breakdown range within and/or beyond which considerable changes may occur in the flow structure which could lead to a negative value of $\partial \mathrm{C}_{\mathrm{L}} / \partial$ $\varphi$.
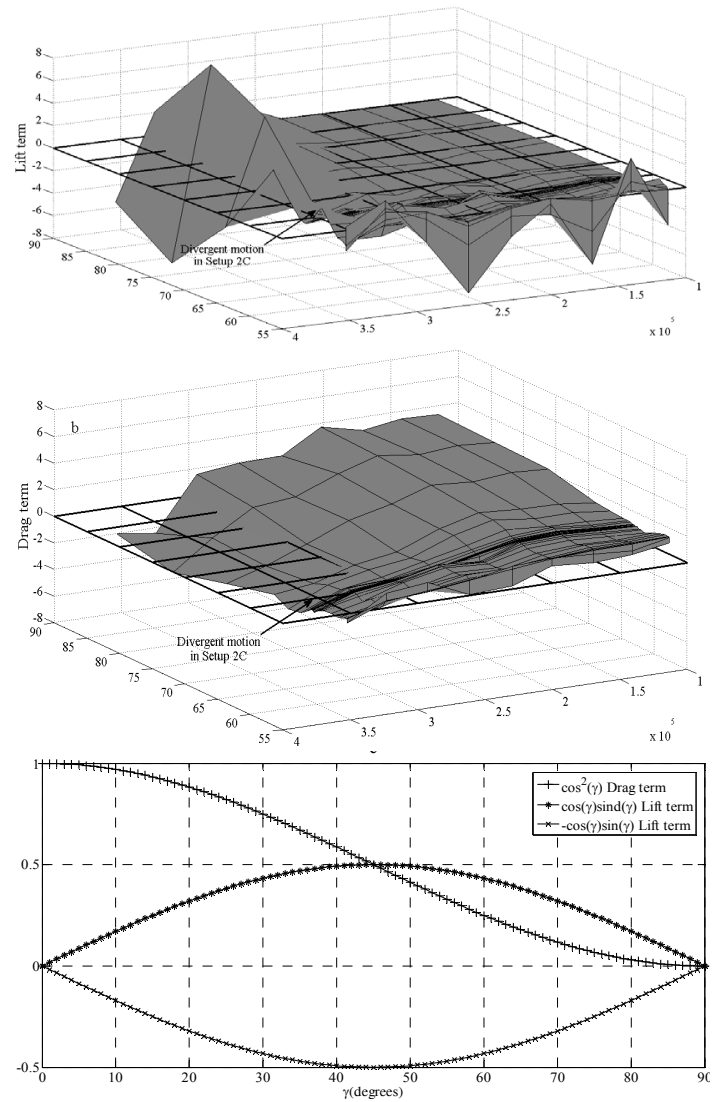

Fig. 6. Contribution of lift and drag terms in total aerodynamic damping; a) Lift term=

$$
\left[C_{L}\left(2 \sin \phi-\frac{1}{\sin \phi}\right)+\frac{\partial C_{L}}{\partial \operatorname{Re}} \operatorname{Re} \sin \phi+\frac{\partial C_{L}}{\partial \phi} \cos \phi\right]
$$

b) Drag term $=$

$$
\left[C_{D}\left(2 \sin \phi+\frac{\tan ^{2} \gamma}{\sin \phi}\right)+\frac{\partial C_{D}}{\partial \operatorname{Re}} \operatorname{Resin} \phi+\frac{\partial C_{D}}{\partial \phi} \cos \phi\right]
$$

c) Weight functions

It is evident from Fig. 4 that in Setup $2 \mathrm{C}$, when $\operatorname{Re}>3 \times 10^{5}$ and $\varphi$ is within this breakdown range, both negative and positive aerodynamic damping ratios could exist. Namely, within this relatively narrow range, the flow is extremely sensitive to the change in the wind-cylinder relative angle where formation or burst of a separation bubbles could occur within the critical regime.

In Eq. (2), the weight functions of the lift and drag terms in the expression of the aerodynamic damping ratio are $\pm \sin (\gamma) \cos (\gamma)$ and $\cos ^{2}(\gamma)$, respectively. To evaluate the contributions of these two terms to the aerodynamic damping ratio when the cylinder vibrates along any arbitrary direction, the lift and drag terms, and the variation of the corresponding weight functions are plotted in Fig. 6 for $\gamma=0^{\circ} \sim 90^{\circ}$, i.e. for a full sweep of possible predominant motion direction from in-plane to out-of-plane. As can be seen from Fig. 6a, the lift term is mostly negative for the range of Reynolds number studied (i.e. $\mathrm{Re}=0.86 \times 10^{5} \sim 3.54 \times 10^{5}$ ). In particular, a significant increase-decrease manner is detected within the range $\varphi=63^{\circ} \sim 81^{\circ}$ when $\operatorname{Re}>3 \times 10^{5}$. This could be due to the random formation of a single separation bubble on one side of the cylinder surface. The direction of lift, and thus the sign of $\mathrm{C}_{\mathrm{L}}$ purely depends on at which side the bubble forms. As the Reynolds number is further increased $\left(\mathrm{Re}=3.55 \times 10^{5}\right)$, the second separation bubble forms on the other side of the cylinder surface. Thus, symmetric distribution of surface pressure reappears. This causes a significant drop in the lift force which is accompanied by a negative $\partial \mathrm{C}_{\mathrm{L}} / \partial \mathrm{Re}$ term.

The drag term, as shown in Fig. 6(b), is always positive within the subcritical Re range. Once the flow enters the critical state, $\partial \mathrm{C}_{\mathrm{D}} / \partial \mathrm{Re}$ term becomes negative for the entire range of $\varphi$ and, the contribution of this term to the negative aerodynamic damping becomes more significant (Fig. 6(b)).

The direction of motion is considered in the total aerodynamic damping ratio as the weight functions of lift and drag terms. They are presented in Fig. 6(c). At $\gamma=0^{\circ}$ (i.e. in-plane motion), the weight function of drag term has its maximum value while that for lift term is zero. This suggests that when the vibration is within the cable plane, negative aerodynamic damping could occur in the critical Re regime. However, to the knowledge of the authors, pure in-plane vibration has never been observed in field and experimental studies. The maximum (or minimum) value of the weight function of the lift term occurs at $\gamma=45^{\circ}$, which equals to 0.5 (or 0.5 ). It is noted from Fig. 6 that when $\gamma=45^{\circ}$, the weight function of the drag term also equals to 0.5 . If both lift and drag terms are negative, their weighted values in Eq. (2) could still be negative, resulting in negative aerodynamic damping ratio. In other words, when $\gamma \approx 45^{\circ}$, vibrations along the in-plane and out-of-plane directions are equally important. The total aerodynamic damping ratio is likely to become negative in the critical Re regime. In the current study, the closest experimental value of $\gamma$ to $45^{\circ}$ was $47.5^{\circ}$, which occurred at $\varphi \approx 58.4^{\circ}$ (Fig. 7).

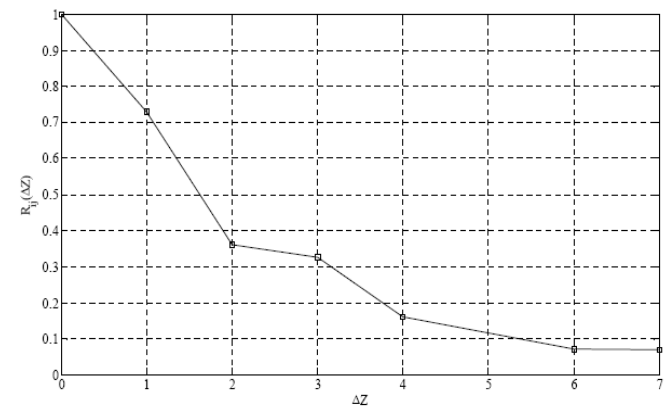

Fig. 7. Correlation coefficients of lift forces calculated at different rings when $\mathrm{Re}=3.55 \times 10^{5}$ under Setup $2 \mathrm{C}$ conditions condition $\left(\phi=60^{\circ}, \gamma=54.7^{\circ}\right)$

This is in fact very close to the critical condition of the divergent response in Setup 2C, where $\varphi=60^{\circ}$ and 
$\gamma=54.7^{\circ}$. However, for out-of-plane vibration, as in the case of Setup 2A, the direction of motion is $\gamma=90^{\circ}$. Therefore, weight functions of both lift and drag terms become zero. In such case, the resulting total aerodynamic damping ratio given by Eq. (3) is always positive.

Macdonald and Larose (2006) also investigated contributions of different terms in Eq. (2) to the cable aerodynamic damping ratio. They pointed out that at $\varphi$ $\approx 60^{\circ}$, the $\partial \mathrm{C}_{\mathrm{L}} / \partial \varphi$ term has the most significant contribution to negative $\zeta_{\mathrm{a}}$ value due to the emergence of critical Re regime. Within the range of $\varphi \approx 65^{\circ} \sim 81^{\circ}$, however, $\partial \mathrm{C}_{\mathrm{L}} / \partial \mathrm{Re}$ and $\partial \mathrm{C}_{\mathrm{D}} / \partial \mathrm{Re}$ contribute more considerably.

It should be noted that, the lift and drag coefficients used in the above analysis are the averaged values of sectional force coefficients sampled at four different longitudinal locations of the cylinder model. They may not express the effects of the instantaneous lift and drag forces. As reported by Cheng et al. (2008b), the divergent type response in the dynamics tests was triggered approximately 100 seconds after $\mathrm{Re}$ is increased to $3.16 \times 10^{5}$. This suggests that instantaneous values of aerodynamic forces and their correlation along the cylinder length are important factors that might contribute to the excitation of unstable motion. The spatial correlation of the instantaneous aerodynamic forces is investigated in the next section.

\subsection{Spatial Correlation}

Spatial correlation of sectional aerodynamic forces is greatly affected by spanwise non-uniformities of flow separation process (Zdravkovich, 1997). Within the lower subcritical Reynolds number range (say up to a couple of thousands), where the flow separation line on the cylinder surface is relatively straight or somewhat wavy but with a very long wavelength, nearly perfect spatial correlation was observed (see, for example, Roshko 1954; Williamson 1989). This is because the transition to the turbulence point is not close enough to the cylinder surface to alter the flow separation process. In the higher subcritical Reynolds number range (Re up to $2 \times 10^{5}$ ), the transition to turbulent flow occurs in the near-wake eddies. The irregular turbulent eddies induce large scale non-uniformity along the separation line, resulting in wavy shape separation line and eddy filaments. These are believed to be responsible for the phase lag between aerodynamic properties along the cylinder axis. In such situations, the straight separation line is disturbed and thus the axial correlation of aerodynamic properties is weakened (Shimizu and Kawamura 1972). Compared to the monotonically decreasing pattern in the subcritical Re range, when entering the critical Re range, the spatial correlation of aerodynamic properties becomes very sensitive to the axial distance between two measurement locations. It has been noted in a few studies that this phenomenon is attributed to the presence of cell-like structures along the span of cylinder in the upper subcritical and critical Re range (Humphreys 1960; Higuchi et al. 1989). In a recent study by Raeesi et al. (2008), it was found that the spatial correlation of sectional aerodynamic forces is considerably enhanced when the two spanwise measurement locations are separated by multiples of cell size.

A parameter which signifies the spanwise uniformity of flow past a cylinder is the correlation length, $\mathrm{L}_{\mathrm{C}}$. It represents the axial range over which the variation of aerodynamic properties at one station are correlated with those at another. It can be defined as (Zdravkovich, 1997):

$L_{C}=\int_{0}^{\infty} R_{i j}(\Delta z) d z$

where $R_{i j}(\Delta z)$ is the spatial correlation between the two measurement points $i$ and $j$ separated by a distance $\Delta z$. In fact, this parameter shows the deviation of flow field from an assumed ideal two-dimensional one, of which the separation line is straight and it oscillates on the cylinder surface and all aerodynamic properties correlate perfectly.

As suggested by Eq. (4), if the correlation coefficient $R_{i j}(\Delta z)$ of a particular aerodynamic property for the given spacing $\Delta z$ is known, mathematically, the correlation length $L_{c}$ of such a property can be computed by integrating the area under the $R_{i j}(\Delta z)$ versus $\Delta z$ curve.

In the present study, using the surface pressure data sampled at $\mathrm{z} / \mathrm{D}=-2,-1,1$, and 2 (Rings $2-5$ as in Fig. 2b), six combinations of spatial distances $\Delta z$ between the two measurement locations are obtained, i.e. $\Delta z / D=1,2,3,4,5$, and 7 . As an example, Fig. 7 shows the relation between the lift correlation coefficient against $\Delta z / D$ at $R e=3.55 \times 10^{5}$ when the model is oriented under Setup 2C. It can be clearly observed from the figure that with the increase of axial spacing between the two measurement points, the correlation of lift at these two locations diminishes quickly. When $\Delta \mathrm{z} / \mathrm{D} \geq 6$, the lift correlation coefficient drops to less than 0.1 , which implies that under the studied physical conditions, the variation pattern of lift force at a given location is almost independent of that at a distance $6 \mathrm{D}$ away along the model axis. Therefore, when calculating the correlation length $L_{c}$, instead of strictly following Eq.(4) to integrate $R_{i j}(\Delta z)$ from $\Delta z$ of 0 to $\infty$, it can be obtained by summing up the area underneath the $R_{i j}(\Delta z)$ versus $\Delta z$ curve between $\Delta z=0$ to $7 \mathrm{D}$ without sacrificing too much accuracy.

The correlation length of sectional lift and drag force coefficients versus the cylinder-wind relative angle $\varphi$ in Setup 2C $\left(\varphi=60^{\circ}, \gamma=54.7^{\circ}\right)$ and Setup 2A $\left(\varphi=60^{\circ}\right.$, $\left.\gamma=90^{\circ}\right)$ are shown in Figs. 8 and 9 respectively. The correlation coefficients are first calculated using timehistories of sectional lift and drag force coefficients at $\mathrm{z} / \mathrm{D}=-5,-1,1$, and 2 (Rings $2-5$ as in Fig. 2a). Then, the area under the $R_{i j}(\Delta z)$ versus $\Delta z$ curve between $\Delta z=0$ to 7D is computed to determine the correlation length $L_{c}$. The second horizontal axis in these two figures represents the strength of axial component of the flow in terms of the ratio between the axial flow speed and the total oncoming flow speed. It equals to $\cos \varphi$.

In Fig. 8a, the correlation length of lift force in static tests including Setup $2 \mathrm{C}$ condition is shown for three 
critical Reynolds numbers of $\operatorname{Re} \approx 2.94 \times 10^{5} \sim 3.55 \times 10^{5}$. At $\operatorname{Re}=3.55 \times 10^{5}$, the correlation length increases significantly with increasing $\varphi$ from $55^{\circ}$ to $60^{\circ}$. The peak at $\varphi=60^{\circ}$ corresponds to the critical condition of Setup $2 \mathrm{C}\left(\varphi=60^{\circ}, \gamma=54.7^{\circ}\right)$. Interestingly, this range coincides with the breakdown range identified earlier $\left(\operatorname{Re}>3 \times 10^{5}, \varphi \approx 55^{\circ}-64^{\circ}\right)$. This suggests that the dramatic changes in the symmetry of flow field surrounding the cylinder are associated with spatially correlated flow patterns around the cylinder. This phenomenon, however, was only observed at $\mathrm{Re}=3.55 \times 10^{5}$, the highest critical Reynolds number tested in Setup 2C condition. In addition, the correlation length $\mathrm{L}_{\mathrm{C}}$ of drag force is plotted for the same range of Re. The observed $\mathrm{L}_{C}$ peak at $\varphi=60^{\circ}$ is evident at $\mathrm{Re}=3.55 \times 10^{5}$, though the value is smaller as compared to the $\mathrm{L}_{\mathrm{C}}$ of lift.
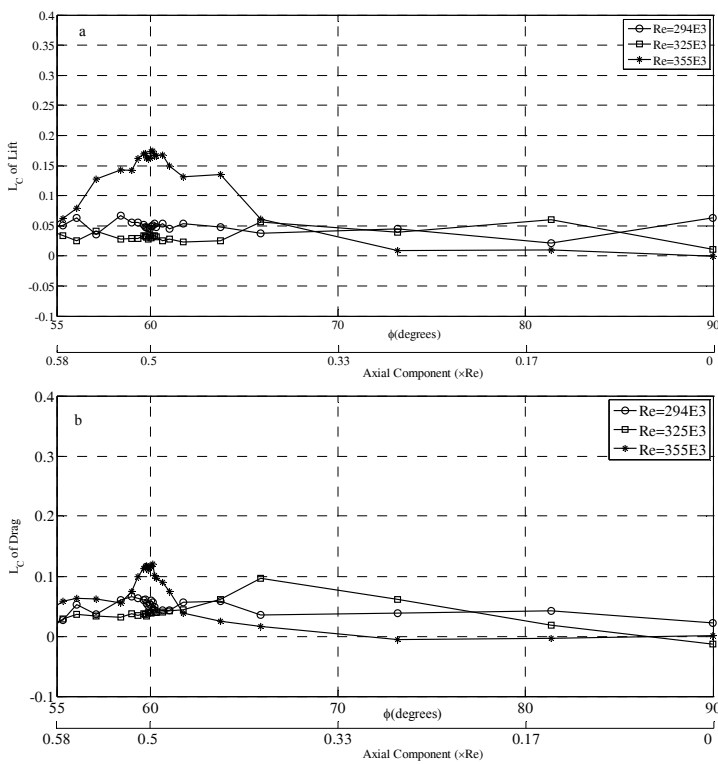

Fig. 8. Correlation length of sectional aerodynamic forces acting on cylinder in the static tests when the model is inclined $54.7^{\circ}$ with respect to the wind, including Setup $2 \mathrm{C}$ condition $\left(\phi=60^{\circ}, \gamma=54.7^{\circ}\right)$; a)

Correlation length of sectional lift force; b) Correlation length of sectional drag force

Large negative and positive values of spatial correlation coefficients within the single separation bubble regime were observed by Raeesi et al. (2008) for cross-flow cylinder $\left(\varphi=90^{\circ}\right)$. These positive and negative values would somewhat cancel out when using Eq. (4) to compute the correlation length. However, when $\varphi=60^{\circ}$, as illustrated in Fig. 8, the enhanced correlation length at critical conditions emerges from the summation of positive correlation values. This could also be interpreted in terms of the deviation from an assumed quasi two-dimensional flow field, i.e. a larger $\mathrm{L}_{c}$ value signifies that the surrounding flow structure agrees more with the ideal quasi two-dimensional flow assumption. In such situation, the maximum sectional instantaneous lift and drag force would occur simultaneously along the span of the cylinder, leading to larger instantaneous overall aerodynamic forces.

Equation (2) shows that negative $\zeta_{\mathrm{a}}$ value of a constant cross-section cylinder in the uniform flow is solely a function of Re, $\gamma$, and $\varphi$. This is because Eq. (2) is derived based on the assumption of quasi-steady aerodynamic forces which are independent of mode shape and modal frequency of the cylinder (Macdonald and Larose, 2006).
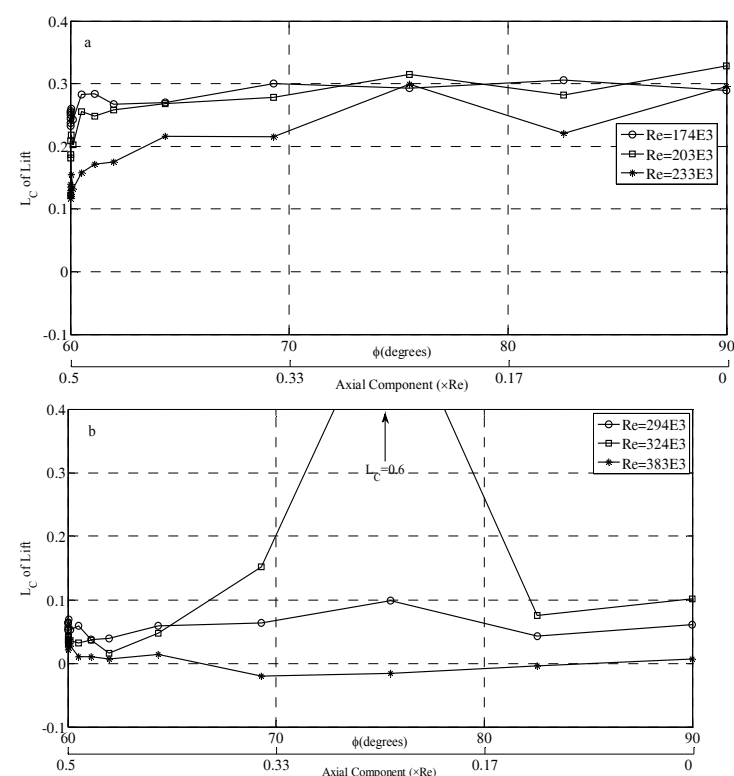

Fig. 9. Correlation length of sectional lift forces acting on cylinder in the static tests when the model is inclined $60^{\circ}$ with respect to the against wind, including Setup 2A

( $\phi=60^{\circ}, \gamma=90^{\circ}$ ) condition; a) Subcritical Reynolds

number range; b) Critical Reynolds number range

Also note that the aerodynamic damping ratio expressed by Eq. (2) is inversely proportional to the natural frequency of the excited mode and thus a mode with lower frequency will yield a larger negative value of $\zeta_{\mathrm{a}}$, which is more likely to excite the cylinder (Macdonald and Larose, 2006). The model used in the current dynamic tests was a segment of stay cable from a real cable-stayed bridge. It was elastically supported at each end by two sets of springs along the in-plane and outof-plane directions. When divergent type of motion occurred, the dominant frequency of the response agreed with the mechanical frequency of the model.

Though site observations (Main and Jones, 2001) show that the wind-induced and rain-wind-induced stay cable vibrations are often in the second, third or even higher modes, due to the limited length of the dynamic model, it is relatively "more rigid" as compared to a full length cable of the same cross section. Thus, it will be difficult to observe the model to be excited in the second or higher modes under the current testing condition. Considering these facts, it appears that a highly correlated flow field along the cylinder, in collaboration with negative aerodynamic damping, might be a determining factor or a necessary onset condition for exciting the divergent motion of a cable in the lower mode. To investigate the observed limited-amplitude response in Setup 2A $\left(\varphi=60^{\circ}, \gamma=90^{\circ}\right)$, the correlation length of sectional lift forces are plotted against the cylinder-wind relative angle $\varphi$, for the Re range of $1.74 \times 10^{5} \sim 3.83 \times 10^{5}$ in Fig. 9. Reynolds number range in Fig. 9(a) falls within the Re range of which the limitedamplitude response was observed in the dynamic tests. 

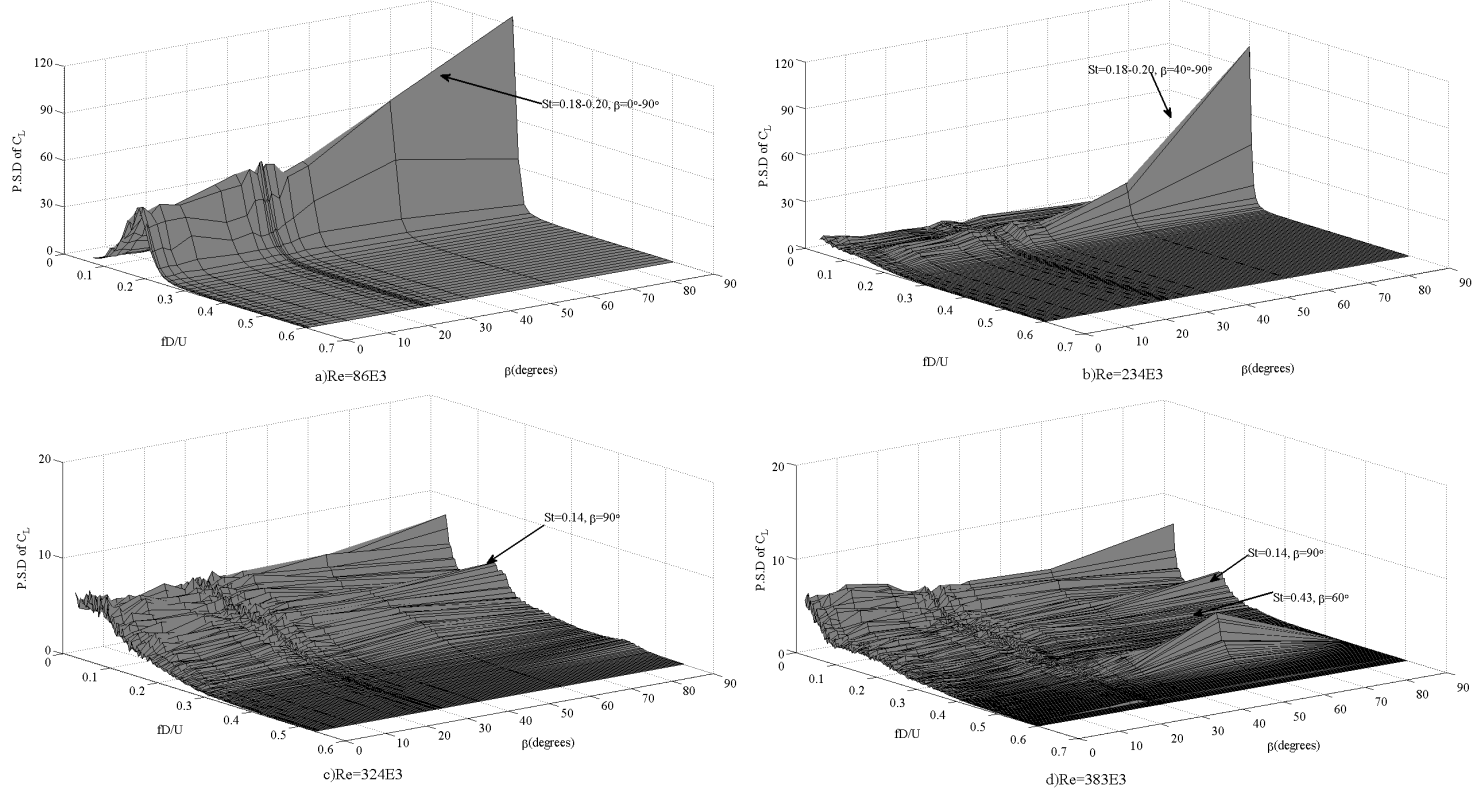

Fig. 10. Power spectra analyses of lift force coefficient at $z / D=$, in the static tests when the model is inclined $54.7^{\circ}$ with respect to the wind, including Setup $2 \mathrm{C}\left(\alpha=54.7^{\circ}, \beta=30^{\circ}, \gamma=54.7^{\circ}\right)$ condition; a) $\left.\operatorname{Re}=0.86 \times 10^{5}, \mathrm{~b}\right) \operatorname{Re}=2.34 \times 10^{5}$, c) $\mathrm{Re}=3.24 \times 10^{5}$, d) $\mathrm{Re}=3.83 \times 10^{5}$

Within this subcritical Re range, the general trend of $\mathrm{L}_{\mathrm{C}}$ typically increases with $\phi$, especially for $\varphi \leq 76^{\circ}$. From $\varphi=60^{\circ}$ to $\varphi=90^{\circ}, \mathrm{L}_{\mathrm{C}}$ increases roughly $70 \%$ for $\mathrm{Re}=2.33 \times 10^{5}$ and about $50 \%$ for $\mathrm{Re}=1.74 \times 10^{5}$ and $2.03 \times 10^{5}$. At $\varphi=90^{\circ}$, the correlation lengths for all three Reynolds numbers are more or less of the same magnitude $\left(\mathrm{L}_{\mathrm{C}} / \mathrm{D} \approx 3.2 \sim 3.5\right)$, while at $\varphi \approx 60^{\circ}, \mathrm{L}_{\mathrm{C}}$ differs more significantly for the three different Reynolds numbers. This indicates that in the subcritical state, the introduction of axial flow could have reduced the spatial correlation and promoted the emergence of critical flow at a Reynolds number lower than that in the cross-flow case $\left(\varphi=90^{\circ}\right)$. The axial flow, which appears to reduce the spatial correlation of flow, is weakened by increasing $\varphi$. In the critical range, however, the axial flow does not alter the correlation length considerably (Fig. 9(b)). Interestingly, a significant increase of correlation length is observed at $\varphi=75^{\circ}\left(\alpha=\beta=60^{\circ}\right)$ when $R e=3.24 \times 10^{5}$. This, and the considerable increase in correlation length observed in Fig. 8 at $\varphi=60^{\circ}$ are perhaps linked with a drastic modification of flow patterns in the critical flow regime when $\operatorname{Re}$ is kept constant but $\varphi$ varies. In addition, as pointed out earlier, the change in wind-cylinder relative angle $\varphi$ would also alter the cylinder cross-sectional shape "seen" by the oncoming flow and thus affect the surrounding flow pattern. Further experimental study is required to confirm and clarify this speculation.

\subsection{Power Spectra Density of Aerodynamic Forces}

The shear-layer instability is responsible for the fluctuating flow field and thus fluctuating lift and drag forces acting on cylinder which cause flow-induced vibrations. Fast Fourier Transform (FFT) is applied to the time-histories of lift and drag forces to obtain their power spectra. In Fig. 10, the Power Spectra Density (PSD) of fluctuating lift force at $\mathrm{z} / \mathrm{D}=1$ (Ring 4 , the ring closest to the mid-span) are shown. The frequency domain is normalized by $U / D$, where $U$ is the free stream wind speed and D is the cylinder diameter. The lift power spectra at four different Reynolds numbers are presented in Fig. 10 from the lowest subcritical $\mathrm{Re}=0.86 \times 10^{5}$, to the highest studied critical Reynolds number of $3.83 \times 10^{5}$.

As can be seen from Figs. 10a and 10b, within the subcritical Re range, for the yaw angles up to $\beta \approx 40^{\circ}$ $\varphi \approx 64^{\circ}$ ), the power spectra energy level does not change considerably with $\beta$. However, for $\beta>40^{\circ}$, the growth in the energy level at the shedding frequency is apparent. At $\operatorname{Re}=2.34 \times 10^{5}$ (Fig. 10b), near the upper limit of subcritical Re range, regular narrow banded vortex shedding occurs only for $\beta>40^{\circ}\left(\phi>64^{\circ}\right)$, more or less at the same energy level as in Fig. 10(a). In contrast, the vortex shedding becomes broad banded when decreasing $\beta$ below 40I, as shown in Fig. 10b.

As expected, when the flow enters the critical regime as depicted in Figs. 10c and 10d, at yaw angle $\beta$ close to $90^{\circ}$, the lift power spectrum loses its dominance and drops almost by an order in magnitude, as compared to those detected in the subcritical Re range shown in Figs. 10a and 10b. This drop occurs to a smaller degree for other yaw angles. The energy becomes more concentrated in the lower frequency range, though minor energy concentration peaks could still be observed at $\mathrm{fD} / \mathrm{U}=0.14 \sim 0.16$ and at $\mathrm{fD} / \mathrm{U}=0.43$ for certain cylinder orientations. Unlike the subcritical case, in the critical range, decreasing yaw angle and hence increasing the intensity of axial flow component does not significantly alter the energy level. As $\beta$ approaches $0^{\circ}$, the power spectra moderately reduce to broadband low energy level.

Since the observed divergent motion is not a purely cross-wind response, the power spectra of drag force are also investigated for the critical regime and are presented in Fig. 11. 


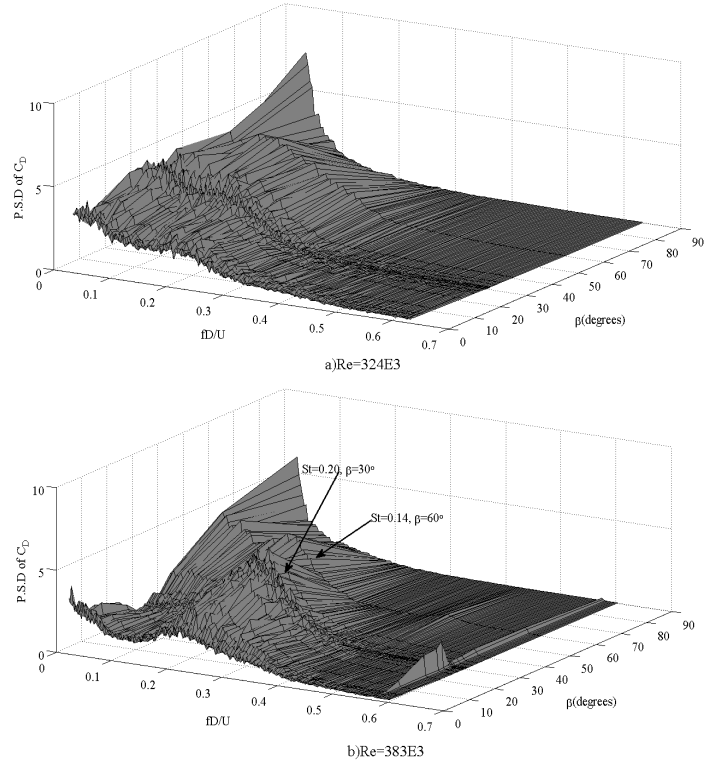

Fig. 11. Power spectra analyses of drag force coefficient at $\mathrm{z} / \mathrm{D}=1$, in the static tests when the model is inclined $54.7^{\circ}$ with respect to the wind, including Setup $2 \mathrm{C}\left(\alpha=54.7^{\circ}, \beta=30^{\circ}, \gamma=54.7^{\circ}\right)$ condition; a) $\left.\mathrm{Re}=3.24 \times 10^{5}, \mathrm{~b}\right) \mathrm{Re}=3.83 \times 10^{5}$

It can be seen that for small yaw angles $\left(\beta<40^{\circ}\right)$, the energy contained in drag has a broadband distribution. In the cross-flow case $\left(\beta=90^{\circ}\right)$, majority of the energy is concentrated in low frequency range without any distinctive peak. This is consistent with the phenomenon observed in the power spectra of lift force in the critical range (Fig. 10d). The flow transition in the boundary layer is accompanied by low frequency intermittent motions, possibly destroying the regular vortex shedding caused by shear layer instability. The low energy, low frequency oscillations of flow field are associated with these intermittent motions in the boundary layer (Higuchi et al. 1989). For the inclined and/or yawed $\left(\beta \neq 90^{\circ}\right)$ case, however, the broadband low energy level oscillations correspond to both axial and circumferential boundary-layer instabilities. It is also interesting to note that at $\mathrm{Re}=3.83 \times 10^{5}$ (Fig. 11b), the highest critical Reynolds number studied, a broadband peak occurs at $\beta=30^{\circ}$, where the frequency corresponding to $\mathrm{St}=0.2$ dominates the energy level.

The power spectra of lift force for the limited-amplitude response observed in Setup 2A are presented in Fig. 12, for the subcritical Re range of $1.73 \times 10^{5}$ to $2.34 \times 10^{5}$ where the unstable motion occurred. It is clear in the figure that for $\beta \leq 40^{\circ} \quad\left(\varphi \approx 60^{\circ} \sim 64^{\circ}\right)$, the lift power spectrum weakens with the increase of Reynolds number. For $\beta 40^{\circ}$, this decrease with respect to increasing $\mathrm{Re}$ within the subcritical $\mathrm{Re}$ range is relatively small. These trends are consistent with the subcritical Re results in Fig. 10. The increased strength of axial flow seems to be partially responsible for the breakup of the regular Karman vortex shedding, i.e. for the wind-cylinder relative angle $\varphi<64^{\circ}$, the normal-tocylinder component of net vorticity vector due to axial boundary-layer becomes important. The net vorticity vector in such situation would not be parallel to the cylinder axis and therefore could weaken the regular shear layer instability caused by the parallel component of vorticity vector. Larger axial flow component seems to interrupt the well defined Karman vortex formation, resulting in the broadening in the power spectra associated with vortex shedding (see, for example, Ramberg, 1983; and Snarski, 2004). It is also revealed in Figs. 10a, 10b, and 12 that as the cylinder yaw angle is swept from $\beta=90^{\circ}$ to $0^{\circ}$, the peak frequency of vortex shedding varies gradually from $\mathrm{St} \approx 0.20$ to 0.17 . This slight shift in the vortex shedding frequency appears to be caused by the same mechanism, that is, the net amount of vorticity discharged to the wake becomes progressively less parallel to the cylinder axis with decreasing $\beta$ (Ramberg 1983). Consequently, the vortex shedding frequency of an inclined circular cylinder would be somewhat less than that in the cross-flow case. Current results are fairly consistent with those by Snarski (2004) within the subcritical range. Snarski identified a Strouhal vortex regime for $\varphi=60^{\circ} \sim 90^{\circ}$, within which the vortex shedding became narrow banded. The narrowband energy distribution pattern representing regular vortex shedding was then replaced by a broadband one for $\varphi=45^{\circ} \sim 60^{\circ}$. This was attributed to a fully separated turbulent flow in the wake of the cylinder.

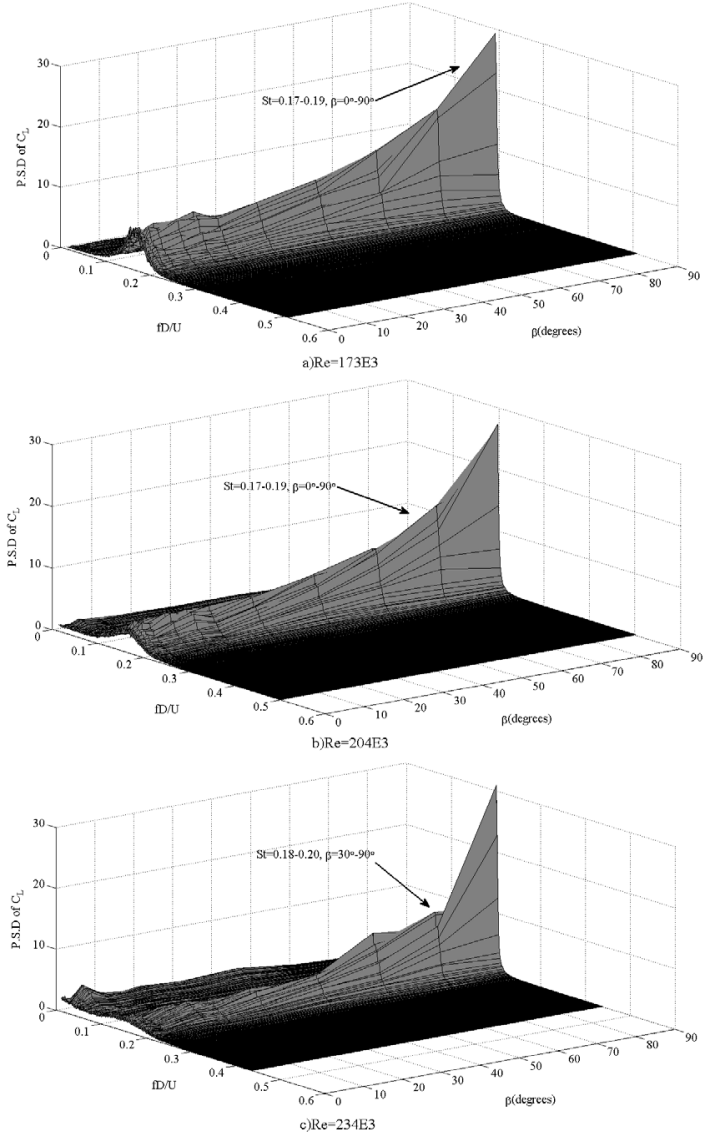

Fig. 12. Power spectra analyses of lift force coefficient at $\mathrm{z} / \mathrm{D}=1$ in the static tests, when the model is inclined $60^{\circ}$ with respect to the wind, including Setup $2 \mathrm{~A}$ $\left(\alpha=60^{\circ}, \beta=0^{\circ}, \gamma=90^{\circ}\right)$ condition; a) $\left.\operatorname{Re}=1.73 \times 10^{5}, b\right)$

$$
\left.\mathrm{Re}=2.04 \times 10^{5}, \mathrm{c}\right) \mathrm{Re}=2.34 \times 10^{5}
$$

Matsumoto et al. (1998, 2007a, and 2007b) noted that the mitigation of Karman vortex shedding, caused by the introduction of axial flow in the subcritical regime, was the major source of the limited-amplitude unstable 
response. This type of response is suppressed at higher Reynolds numbers, as observed by Cheng et al. (2008b). This is associated with the fact that within the subcritical regime, the fluctuations in the separation angle become relatively large just before reaching the transitional Reynolds number range. This would be accompanied by large fluctuations of lift force (Raeesi et al. 2008) within this Re range. Therefore, although the interaction between the flow fields on both sides of the cylinder is discouraged because of the mitigation of regular Karman vortex shedding by the axial flow, the existence of large fluctuation lift component, along with the highly correlated spatial flow structure within the subcritical range as shown in Fig. 9, could still induce the limited-amplitude unstable response or the so-called high-speed vortex excitation observed in Setup 2A $\left(\varphi=60^{\circ}, \quad \gamma=90^{\circ}, \quad \operatorname{Re} \approx 1.86 \times 10^{5} \sim 1.96 \times 10^{5}\right)$. However, when entering the transitional and the critical range, as can be observed from Figs. 9 and 12, the magnitude of both fluctuating lift and correlation length drop considerably, leading to the suppression of the unstable response.

\section{CONCLUding REMARKS}

The possible excitation mechanisms associated with two different types of wind-induced cable vibrations have been investigated based on a set of wind tunnel experiment data on a rigid circular cylinder model. The unsteady surface pressure sampled at four different spanwise locations of a static circular cylinder model were utilized to compute the aerodynamic damping ratio, spatial correlation and power spectra of sectional aerodynamic forces to reveal possible mechanisms of the observed divergent galloping type instability and limited amplitude instability.

Results show that these two types of cable response are triggered by different mechanisms. Within the parameter ranges where these instabilities occurred, the aerodynamic damping ratio of the former has been detected to be negative and thus leads to the continuous growth of response amplitude, whereas that of the latter remains positive.

More interestingly, when investigating the possible mechanisms associated with the galloping type motion, a vortex breakdown range has been identified when the cable-wind relative angle $\varphi$ varies from $55^{\circ}$ to $64^{\circ}$, beyond which remarkable changes in the symmetry and characteristics of flow field would occur. When reaching the critical Reynolds number, the formation and bursting of a single flow separation bubble on one side of the cylinder surface within this vortex breakdown range will drastically alter the symmetry of the surrounding flow pattern. Therefore, a non-zero lift will be generated. And as the relative angle $\varphi$ between the wind and the cable decreases, the magnitude of the lift is observed to increase, which consequently leads to a considerable negative value of $\partial \mathrm{C}_{\mathrm{L}} / \partial \varphi$ term. This effect, along with the negative values of $\partial \mathrm{C}_{\mathrm{L}} / \partial \mathrm{Re}$ and $\partial \mathrm{C}_{\mathrm{D}} / \partial \mathrm{Re}$ terms in the critical Re range was proposed to be of great importance for the negative aerodynamic damping ratio when $\mathrm{Re}>3 \times 10^{5}$. For the range of $\varphi \approx 65^{\circ} \sim 81^{\circ}$, however, it seems that $\partial \mathrm{C}_{\mathrm{L}} / \partial \mathrm{Re}$ and $\partial \mathrm{C}_{\mathrm{D}} / \partial \mathrm{Re}$ terms have major contributions to the negative aerodynamic damping ratio. Further experiments are required to verify the possible occurrence of divergent motion under this set of physical conditions.

In addition to the negative aerodynamic damping ratios $\zeta_{\mathrm{a}}$, when the divergent response occurred, the spatial flow structure is found to be highly correlated along the cylinder span. This suggests that the collaboration of sectional aerodynamic forces along the cylinder axis could be an important or even a necessary factor for the excitation of cable vibrations on real bridges.

On the other hand, the limited-amplitude instability is found to be associated with the mitigation of regular Karman vortex shedding in the breakdown range while the spatial flow field was highly correlated. This instability is suppressed when reaching the critical Re range owing to the decrease of the correlation length.

\section{ACKNOWLEDGEMENTS}

The present work was made possible by support of Natural Sciences and Engineering Research Council of Canada (NSERC). We are also grateful to National Research Council of Canada (NRCC).

\section{REFERENCES}

Bosch, H. (2006). Review of bridge cable vibrations within the USA. Proceeding of 2006 Wind Induced Vibration of Cable Stay Bridges Workshop (on CD-ROM).

Cheng, S., P.A. Irwin, and H. Tanaka (2008a). Experimental study on the wind-induced vibration of a dry inclined cable-Part II: proposed mechanism. J. Wind Eng. Ind. Aerodyn. 96, 22542272.

Cheng, S., G.L. Larose, M.G. Savage, H. Tanaka, and P.A. Irwin (2008b). Experimental study on the wind-induced vibration of a dry inclined cablePart I: Phenomena. J. Wind Eng. Ind. Aerodyn. 96, 2231-2253.

Cheng, S., H. Tanaka (2005). Correlation of aerodynamic forces on an inclined circular cylinder. Wind and Structures an Int. J. 8(2), 135146.

Cheng, S., H. Tanaka, G.L. Larose and M.G. Savage (2003). Aerodynamic behaviour of an inclined circular cylinder. Wind and Structures an Int. J. 6(3), 197-208.

Den Hartog, J.P. (1956). Mechanical Vibrations (fourth $E d$.), McGraw-Hill, New York.

Higuchi, H., H.J. Kim and C. Farell (1989). On flow separation and reattachment around a circular cylinder at critical Reynolds numbers. J. Fluid Mech. 200, 149-171.

Hikami, Y. N. Shiraishi (1988). Rain-wind induced vibration of cables in cable-stayed bridges. J. Wind Eng. Ind. Aerodyn. 29, 409-418. 
Honda, A., T. Yamanaka, T. Fujiwara, and T. Saito (1995). Wind tunnel test on rain-induced vibration of the stay cable. Proceedings of International Symposium on Cable Dynamics, Lie'ge, Belgium, 255-262.

Humphreys, J.S. (1960). On a circular cylinder in a steady wind at transition Reynolds numbers. $J$. Fluid Mech. 9, 603-612.

Irwin, P.A., A. Nedim, and N. Telang (1999). Wind induced stay cable vibrations a case study. Proceeding of the Third International Symposium on Cable Aerodynamics, Trondheim, Norway, 171-176.

Macdonald, J.H.G., and G.L. Larose (2006). A unified approach to aerodynamic damping and lift/drag instabilities and its application to dry inclined cable galloping. J. of Fluids and Structures 22, 229-252.

Main, J.A., and N.P. Jones (2001). Evaluation of viscous dampers for stay-cable vibration mitigation. J. of Bridge Eng. ASCE. 6 (6), 385397.

Matsumoto, M., T. Yagi, Y. Adachi, H. Hatsuda, and T. Shima (2007a). Cross flow response of circular cylinders influenced by Karman vortex mitigation. Proceedings of Seventh International Symposium on Cable Dynamics, Vienna, Austria.

Matsumoto, M., T. Yagi, Y. Adachi, H. Hatsuda, and T. Shima (2007b). Sensitivity of dry-state galloping of cabled stayed bridges to Scruton number. Proceedings of Seventh International Symposium on Cable Dynamics, Vienna, Austria.

Matsumoto, M., T. Yagi, and D. Tsushima (1999). Vortex-induced vibration of inclined cables at high wind velocity. Proceedings of the 10th International Conference on Wind Engineering, Copenhagen, Denmark, 979-986.

Matsumoto, M. (1998). Observed behavior of prototype cable vibration and its generation mechanism. Bridge Aerodynamics, Larsen \& Esdahl (eds), Balkema, Rotterdam, 189-211.

Matsumoto, M., N. Shiraishi, M. Kitazawa, C. Knisely, H. Shirato, Y. Kim, and M. Tsujii (1990). Aerodynamic behaviour of inclined circular cylinders-cable aerodynamics. J. of Wind Eng. and Ind. Aero. 33, 63-72.

Miyata, T., H. Yamada, and T. Hojo (1994). Aerodynamic response of PE stay cables with pattern-indented surface. Proceedings of International Conference on Cable-Stayed and Suspension Bridges (AFPC), Deauville, France 2, 515-522.

Raeesi, A., S. Cheng, and D.S.K. Ting (2008). Spatial flow structure around a smooth circular cylinder in the critical Reynolds number regime under crossflow condition. Wind and Structures, an Int. J. 11(3), 221-240.

Ramberg, S. E., (1983). The effects of yaw and finite length upon the vortex wakes of stationary and vibrating cylinders. J. Fluid Mech. 128, 81-107.

Roshko, A. (1954). On the development of turbulent wakes from vortex streets. NACA Report No., TR1191.

Saito, T., M. Matsumoto, and M. Kitazawa (1994). Rain-wind excitation of cables of cable-stayed Higashi-Kobe Bridge and cable vibration control. Proceedings of International Conference on Cable-Stayed and Suspension Bridges (AFPC), Deauville, France 2, 507-514.

Schewe, G. (1983). On the force fluctuations acting on a circular cylinder in cross-flow sub-critical up to transcritical Reynolds numbers. J. Fluid Mech. 133, 265-285.

Shimizu, K. and M. Kawamura, M. (1972). Spanwise correlation measurement behind a circular cylinder in subcritical Reynolds number region. J. Phys. Soc. Jpn. 32, 1454-1454.

Shirakashi, M., A. Hasegawa and S. Wakiya (1986). Effect of the secondary flow on Karman vortex shedding. Bulletin of JSME. 29, No. 250.

Snarski, S. R. (2004). Flow over yawed circular cylinders: Wall pressure spectra and flow regimes. Phys. of Fluids 16(2), 344-359.

Virlogeux, M. (1998). Cable vibrations in cable-stayed bridges. In: Bridge Aerodynamics, Balkema, 213233.

Williamson, C.H.K. (1997). Advances in our understanding of vortex dynamics in bluff body wakes. J. of wind Eng. And Ind. Aerodyn. 69-71, 3-32.

Yagi, T., H. Naito, Z. Liang, and H. Shirato (2009). Evaluation of aerodynamic forces on an inclined cable in consideration of end conditions of model for wind tunnel tests. Proceedings of 8th International Symposium on Cable Dynamics, Paris, France, 151-158.

Zdravkovich, M.M. (1997). Flow around circular cylinders: Fundamentals (Vol. 1), University Press, Oxford, England.

Zuo, D. and N.P. Jones (2009). Wind tunnel testing of yawed and inclined circular cylinders in the context of field observations of stay cable vibrations. J. of wind Eng. And Ind. Aerodyn. 97, 219-227. 